\title{
Finish Line or Beginning? Welcome the New Board-certified Surgical Oncologists
}

\author{
Douglas S. Tyler, $\mathrm{MD}^{1,2}$ and Fabrizio Michelassi, $\mathrm{MD}^{3}$ \\ ${ }^{1}$ Department of Surgery, University of Texas Medical Branch, Galveston, TX; ${ }^{2}$ VA Medical Center, Durham, NC; \\ ${ }^{3}$ Department of Surgery, Weill Cornell Medicine, New York, NY
}

The application by the American Board of Surgery (ABS) to the American Board of Medical Specialties (ABMS) for a subspecialty certificate in Complex General Surgical Oncology (CGSO) was unanimously approved by the ABMS Board of Directors and ratified by the ABMS Assembly in March 2011. In February 2015, the ABS conferred this certificate to the first 56 candidates who successfully passed both the first Qualifying Exam (QE) and Certifying Exam (CE). This editorial reviews the events that occurred during those intervening 4 years and adds to the previous publications that have chronicled the road traveled by our specialty from the very first efforts at a certificate 30 years ago. ${ }^{1-3}$

\section{DEVELOPMENT OF CGSO CE}

The creation of a multiple-choice question bank sufficient for at least two separate and different QEs took an enormous amount of work by 12 consultants to the Surgical Oncology Board from fall 2011 to spring 2012. ${ }^{3}$ Seventyfour individuals took the CGSO QE in September 2014, and the CGSO Board met on September 30 in Philadelphia with ABS psychometricians to set the passing score for this examination. At that meeting, the passing grade was set at $74 \%$ correct. The mean score was $78.3 \%$ correct with a standard deviation of $4.3 \%$. Seven of the 74 individuals did not receive a passing score, for a pass rate of $90.5 \%$. The failure rate was $9.5 \%$.

\footnotetext{
(C) Society of Surgical Oncology 2016

First Received: 19 January 2016; Published Online: 24 February 2016

F. Michelassi, MD

e-mail: fam2006@med.cornell.edu
}

Concurrent with the development of the $\mathrm{QE}$, a significant amount of work was necessary to develop content and case scenarios for the CE. The CGSO Board had to initially decide what the structure of the CE should be before developing the content for the examination. After much discussion, the CGSO board moved forward with a modular format where there would be five oral examination rooms to test examinees on content focused in specific areas. The five areas were as follows: endocrine/head and neck/upper gastrointestinal (GI); hepatobiliary and pancreatic; colorectal and small bowel; breast/genetics; and melanoma/sarcoma/clinical trials.

A decision was then made to follow an examination format similar to the ABS General Surgery CE. In each room, the examinee would be asked four questions over a 30 -min period. There would be two examiners. Each examiner would be an expert in the content of the room they would be examining in, but interestingly none of the examiners would be board certified in Complex General Surgical Oncology. A decision to have no grandfathering for this new certificate was made back in 2009, when the Society of Surgical Oncology (SSO) Executive Committee unanimously voted against granting certificates to individuals who had not completed surgical oncology fellowships in Accreditation Council for Graduate Medical Education (ACGME)-approved programs. The scoring format would be similar to the current ABS structure for the CE in General Surgery, where individuals would be scored on a scale of 4-6. Scores could not be changed after the examiners completed their testing.

Working closely with the ABS leadership, the CGSO Board determined that they would need a pool of 40 questions for each of the five content areas. To build the bank of CE questions, a second group of consultants was pulled together who helped over the next several months to build the CE question pool to contain the 40 required CE 
questions within each of the five broad testing areas. The individuals who contributed to the development of CE case scenarios are listed in Table 1. Case scenarios, once created, were then vetted by the Complex Surgical Oncology Board members so that they could be finalized and formatted in a uniform fashion for entry into the examination book.

With the first QE being offered in fall 2014, the next challenge was setting a time and place for the CE. This proved surprisingly difficult, as a time was needed when examiners could give four consecutive days to the examination process. Picking a time in late winter/early spring was further complicated by the large number of surgical meetings that are already scheduled in this time period, as well as by school spring breaks, for which many examiners had vacation time planned. Ultimately, the window identified with the fewest conflicts and the easiest access was for the CE to be held February 8-11, 2015, in Philadelphia.
The team of examiners who volunteered to participate is shown in Fig. 1 and listed in Table 2.

The examination teams assembled in Philadelphia on February 8, 2015. A preexamination orientation was held to discuss and review the general format of the CGSO CE examination process. Fortunately, about a third of the examiners were current or former ABS directors and were well versed in the CE process. Additionally, most of the other examiners had participated as associate examiners in at least one ABS CE for general surgery. The specifics of daily examinations was carried out each morning before the examinations started, when the examiners would meet over breakfast to select cases for the day from each topic area. The days were broken into a morning session and an afternoon session. A session would consist of five 30-min blocks where the examinee would be examined by two examiners in each of the five disease areas (breast, hepatobiliary, colorectal, melanoma/sarcoma, and stomach/endocrine). There were

TABLE 1 People who helped create clinical scenarios for first CGSO Board CE

\begin{tabular}{ll}
\hline Scenario & People \\
\hline $\begin{array}{l}\text { Melanoma/sarcoma/peritoneal } \\
\text { malignancy/clinical trials }\end{array}$ & Doug Tyler, Jeff Gershenwald, Russ Berman, Vern Sondak, Richard Alexander, Todd Tuttle, Sam \\
Yoon & Mitch Posner, Tony Senagore, Alessandro Fichera, Alan Herline, Marty Heslin, Matt Kalady, Miguel \\
& Rodriguez-Bigas, Marty Weiser \\
Chris McHenry, Margo Shoup, Mark Allen, Gerry Doherty, Herb Chen, James Howe, Martin Karpeh, & Geoff Thompson \\
Breast & Peter Beitsch, Suzanne Klimberg, Kelly Hunt, Shelley Hwang, Brian Kaplan, Walton Taylor, Kim \\
& Van Zee \\
Hepatobiliary/pancreatic & Selywn Vickers, Reid Adams, Mike Choti, Bryan Clary, Jason Flemming, Andrew Lowy, Sharon \\
& Weber, Charles Vollmer
\end{tabular}

CGSO Complex General Surgical Oncology, CE certifying examination

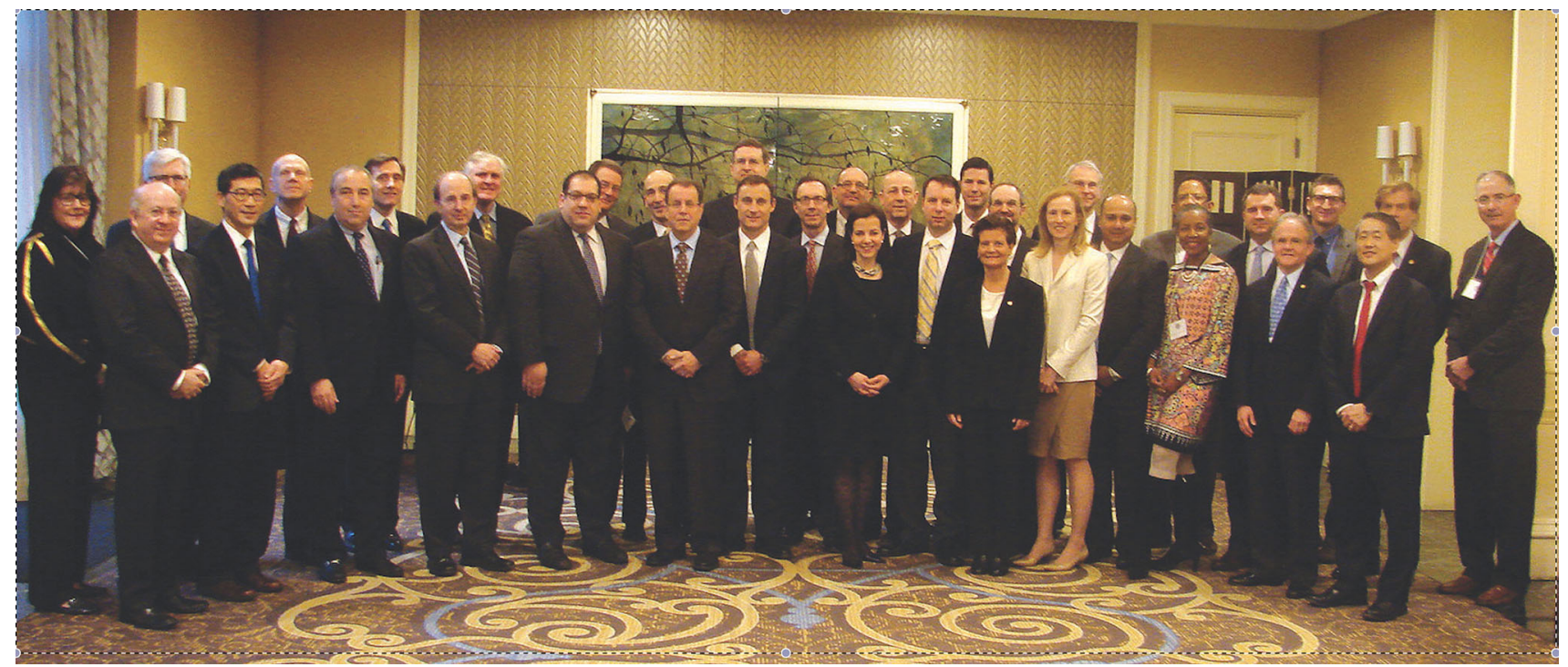

FIG. 1 Picture of the examiners at the inaugural Complex Surgical Oncology Board Certifying exam in Philidelphia. February 8-11, 2015 
TABLE 2 Examiners by specialty and room assignment for first CGSO Board CE

\begin{tabular}{|c|c|}
\hline Specialty & Examiner \\
\hline Breast & Suzanne Klimberg, Nataline Johnson, Peter Beitsch, Kelly Hunt, Ari Brooks, Ron Weigel \\
\hline Hepatobiliary/pancreatic & Selwyn Vickers, Reid Adams, Mike Choti, Doug Evans, Ron Dematteo, Ken Tanabe, Jeff Drebin \\
\hline $\begin{array}{l}\text { Stomach/endocrine/ } \\
\text { thoracic }\end{array}$ & Chris McHenry, Cam Wright, Gerry Doherty, John Olson, Herb Chen, Douglas Fraker, Nancy Perrier, Rachel Kelz \\
\hline Melanoma/sarcoma & $\begin{array}{l}\text { Douglas Tyler, Margo Shoupe, Russell Berman, Vernon Sondak, Merrick Ross, Kelly McMasters, Jeffery } \\
\text { Gershenwald }\end{array}$ \\
\hline Colorectal & Mitch Posner, Jim Fleshman, Martin Weiser, Matt Kalady, Howard Ross, Mark Evers, Tony Senagore \\
\hline
\end{tabular}

CGSO Complex General Surgical Oncology, $C E$ certifying examination

TABLE 3 Scoring definitions for CGSO CE

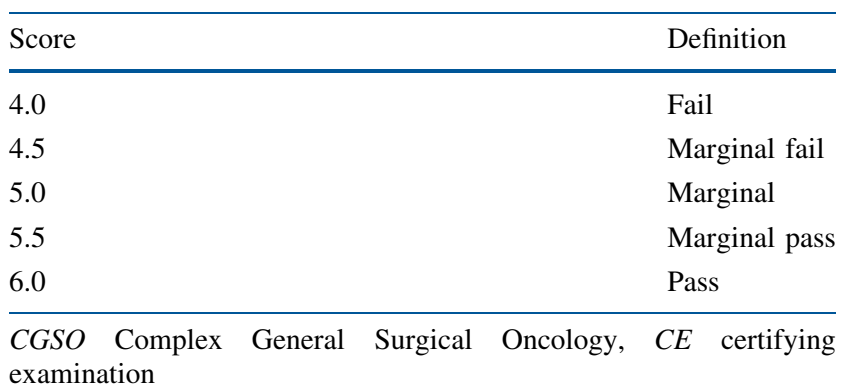

four questions per room scored on a scale of 4/4.5/5/5.5/6 (Table 3). Each examiner would score the candidate. At the end of each session, the scores of each of the 10 examiners who tested the candidate would be added together. A passing grade was determined to be 52.5 or greater. There was no postscoring modification for individual examiner variability. All examinees in a given exam session would have the same questions. Questions used in the morning session would not be used in any other session. Once each disease group picked questions for a given morning or afternoon session, they would be discussed in front of the other groups to make sure that overlapping questions were not being given, such as a question about a GI stromal tumor being given in both the sarcoma room and the stomach/endocrine/thoracic room.

Sixty-three candidates took the CGSO CE on February 9-11, 2015. Fifty-six of the 63 candidates passed the examination and have been certified in Complex General Surgical Oncology. The failure rate was (7/63) $11.1 \%$. This failure rate is similar to other specialty boards. In total, 74 individuals took the CGSO QE, and ultimately 56 passed the CGSO CE, for a $76 \%$ certification rate.

\section{DEVELOPMENT OF MILESTONES FOR FELLOWS IN CGSO TRAINING PROGRAMS APPROVED BY ACGME}

Concurrent with the development of the CGSO CE was the development of milestones for fellows in Complex General Surgical Oncology programs. This process was initially carried out at a retreat in Chicago on January 19-20, 2014. The working group at the retreat was chaired by Danny Takanishi and Laura Edgar. Others participating in the retreat included Russell Berman, Chris McHenry, Paula Termuhlen, Douglas Tyler, and Peggy Simpson. The retreat had as an advisory group of Residency Review Committee-Surgery (RRC-S) members James Herbert, Lenworth Jacobs, and John Potts III. The goal of the group was to develop milestones for complex general surgical oncologist in the six areas of competency that included the following: patient care (evaluations and multimodal care, technical aspects of surgery); medical knowledge; systems-based practice; practice-based learning and improvement; professionalism; and interpersonal and communication skills. The milestones would allow tracking of the progress of fellows from level 1 , which would equate to the skills that should be present in an incoming fellow, to level 5 , which would equate to the level that fellows should aspire to. A representative example of levels that would track progression through milestones in the patient care category of hepatobiliary surgery is shown in Fig. 2. Although fellows should ideally aspire to the highest level, a level of 4 would denote a level of competency that would be sufficient for graduation. The Clinical Competency Committee, which comprises three or more active teaching faculty from the fellowship who advise the program director, would then meet twice a year to review each fellow's progress at a given institution using the milestones. The milestones would allow programs to promote fellows based on achieving predefined competencies in place of the current time-based framework for progression in traditional fellowships. Feedback from these meetings would then be given to the fellows so they could work to optimize their performance and training.

\section{REEVALUATION OF CASE NUMBERS IN LINE WITH GOAL FOR CREATING ABS CERTIFICATE IN CGSO}

Before the ACGME approval process, surgical oncology fellowships were evaluated and approved by the SSO. The 


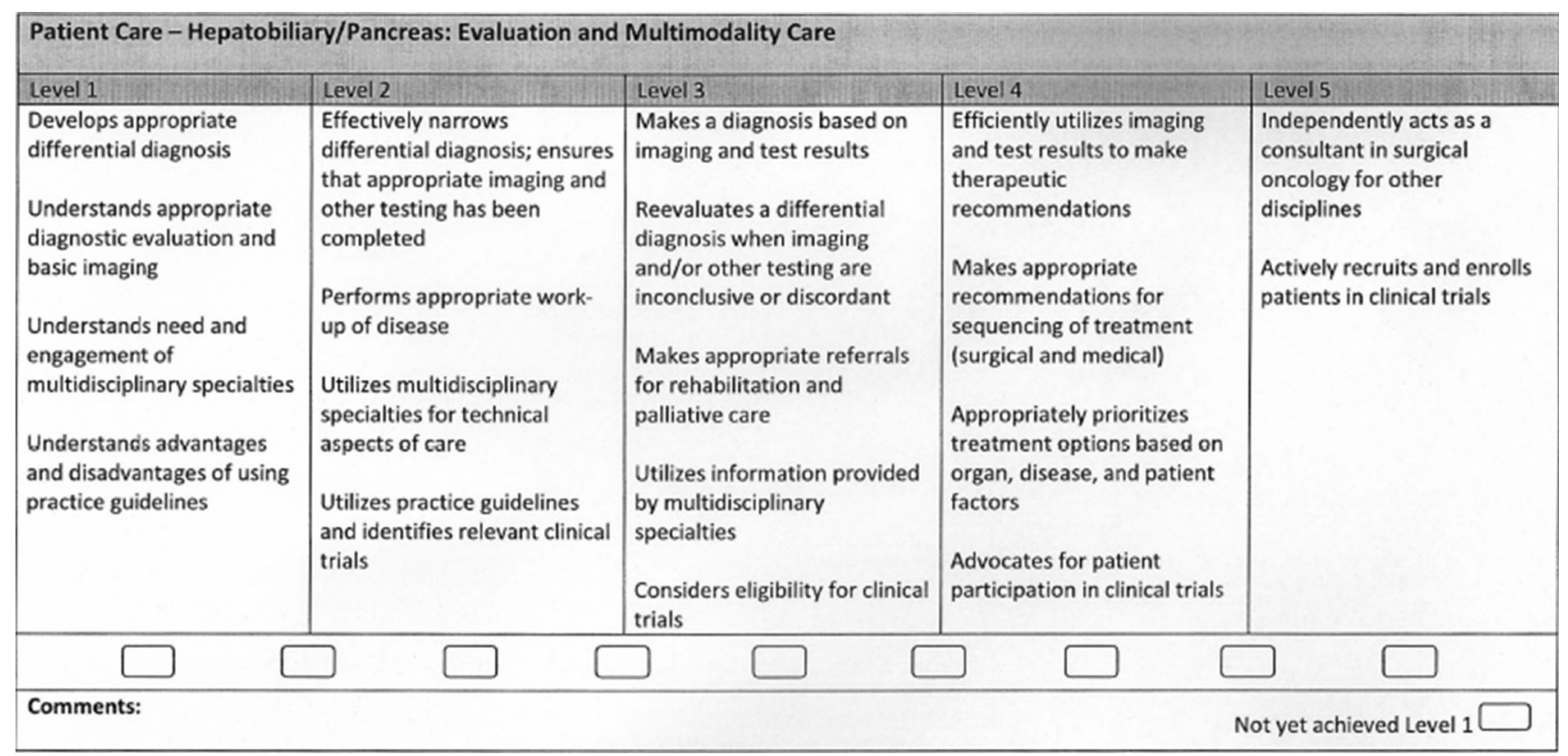

FIG. 2 Milestones broken down by levels for the competency of Patient Care in the Area of hepatobiliary/pancreas surgery

SSO had a requirement that to stay in good standing, finishing fellows should perform a minimum of 150 cases. At the time the ACGME initially approved the 16 existing United States-based SSO-approved surgical oncology programs in 2013, the number of cases that graduating fellows were required to have before sitting for the QE was kept the same. The CGSO and the ABS questioned whether this number was appropriate for the new certificate. This number was based on a 2001 recommendation that SSOapproved surgical oncology fellowships have a minimum of 150 cases, with additional case list requirements to include a minimum of 35 breast cases, 10 melanoma cases, 10 regional lymphadenectomies, five sarcomas, nine endocrine, 15 hepatopancreatobiliary, 15 colorectal, and three thoracic.

In 2011, the SSO revisited the issue of minimum case numbers by creating an SSO Training Committee Task Force to help develop a recommendation on this issue. The goal was to define a set of minimum case requirements in each of the major disease sites for surgical oncology fellowship training. These proposed requirements were structured to maximize the educational value of the fellowship training programs and to facilitate the acquisition of competency across a broad range of disease processes. In theory, they would provide benchmarks to achieve a balanced 2-year clinical curriculum. The Task Force obtained 2-5 years of historical case log data from surgical oncology fellowship programs (Partners, Toronto, VCU [incomplete], OSU, RWMC, MSKCC, MDACC, JHU, UCMC, Pittsburgh, Miami, University of Chicago) and one nonapproved fellowship program (UNC). No data were submitted from seven programs (City of Hope, Fox Chase, Moffitt, John Wayne, McGill, Roswell Park, Calgary, Louisville). Using this historical data, the Task Force was able to determine problematic areas, estimate the current fellowship's ability to meet these requirements, and set the groundwork for a validation study over the next 2 years in all SSO surgical oncology fellowships using the newly designed SSO case log system.

There are several pitfalls with this data set, including the fact that data were collected using the ACGME case logs, which allowed fellows to designate only one case for credit. Therefore, certain fellow case logs may record one case but not a defined linked procedure (e.g., mastectomy + axillary resection only were scored as mastectomy). In addition, some of the fellowship programs reported incomplete data or averages of their fellowship over a specified time period. Finally, certain cases were difficult to adequately quantify (palliative procedures, wedge gastrectomy vs. subtotal gastrectomy, retroperitoneal and extremity sarcomas, disease process associated with radical lymphadenectomy procedure). Despite these limitations, the group proposed a new case number for fellows (Table 4).

In the process of multiple SSO-approved programs transitioning to ACGME-approved programs in 20122013, the Task Force recommendations were never implemented because oversight of these fellowships transitioned from the SSO to the ACGME and the newly formed CGSO Board. The ABS, ACGME, and the CGSO Board discussed revisiting the minimum case numbers required for fellows to sit for the $\mathrm{QE}$ and decided to 
TABLE 4 Proposed 2011 SSO Training Committee minimum case requirement recommendations for fellows

\begin{tabular}{lc}
\hline Disease site & $\begin{array}{l}\text { No. operations or } \\
\text { cases }\end{array}$ \\
\hline Breast & 60 \\
Melanoma and unusual cutaneous malignancies & 18 \\
Regional lymphadenectomy (major, exclusive & 19 \\
$\quad$ of breast) & \\
Sarcoma & 15 \\
Endocrine & 21 \\
Regional therapies & 5 \\
Upper gastrointestinal & 14 \\
Hepatobiliary & 22 \\
Pancreas/duodenal/periampullary & 17 \\
Colorectal & 42 \\
Genitourinary & 2 \\
Gynecologic oncology & 2 \\
Surgical management of advanced & 10 \\
$\quad$ cancers/palliative care & \\
Total & 239 \\
Surgical pathology cases & 27 \\
Medical oncology cases & 25 \\
Radiation oncology cases & 15 \\
Prevention and community outreach cases & 3 \\
Cancer-related rehabilitation/genetic counseling & 8 \\
$\quad$ cases & \\
\hline
\end{tabular}

SSO Society of Surgical Oncology

examine the first 2 years of fellows now completing ACGME-approved Complex General Surgical Oncology programs in 2013 and 2014. The CGSO Board (which has representation of multiple surgical organizations that involve cancer management, including the SSO, Americas
Hepato-Pancreato-Biliary Association, American Association of Endocrine Surgeons, American Society of Breast Surgeons, and ABS) generally agreed with the SSO Training Committee Task Force recommendations with slight modifications. First, the CGSO Board rounded the case numbers up to 240 and thought that the case list distribution should be more generally lumped together by disease site with defined minimums, as shown in Table 5 . The CGSO Board also provided more clear-cut definitions for the case numbers, as follows:

(1) Oncologic cases are defined as those involving neoplastic diseases, performed for a presumptive or known diagnosis of cancer, involving premalignant processes, and/or carried out for cancer prevention.

(2) Fellows can be either a primary surgeon or a teaching assistant on surgical cases.

(3) Multidisciplinary cases are defined as patient case discussions in a multidisciplinary forum involving surgical and nonsurgical clinical colleagues regarding patients with whom the fellow is involved in their clinical care. The fellow must be present at the forum in which the case is discussed to be able to count the case. The clinical encounter does not have to involve a surgical procedure. Multidisciplinary cases that do involve a surgical procedure can also be counted as surgical case in addition to a multidisciplinary case.

The CGSO Board recommended some minimum numbers of surgical cases in broadly defined categories to make sure there was diverse clinical exposure available in the CGSO programs, as highlighted in Table 5. The number was kept at a lower level to allow for tracking in areas that may be of interest to the candidate, as long as the 240 case minimum was reached. There would be no maximum case

TABLE 5 Recommendation of case list by CGSO Board

\begin{tabular}{lll}
\hline Oncologic area & Minimum no. cases & Multidisciplinary cases \\
\hline Breast & 40 & 25 \\
Hepatobiliary/pancreatic & 35 & 25 \\
Non-HPB GI & 50 & 25 \\
Endocrine & 15 & 15 \\
Melanoma/soft tissue sarcoma & 30 & 30 \\
& 170 New minimum total & 120 \\
& Additional 70 above these minimums & \\
Total cases & have to broadly fit into SO-SCORE categories & 120 \\
Multidisciplinary management & 240 & Can have overlap of surgical cases and \\
\multicolumn{1}{l}{ experiences } & 120 with subcategorization as shown above & cases documenting multidisciplinary \\
& & management; presents at conference
\end{tabular}

CGSO Complex General Surgical Oncology, HPB hepatobiliary, GI gastrointestinal, SO-SCORE Surgical Oncology-Surgical Council on resident education 
limits, but this will be revisited in 2 or 3 years to determine if a maximum number of cases in each category should be considered.

The CGSO Board also recommended increasing to 120 the number of multidisciplinary cases (from 78 recommended by the SSO Task Force), where patient management is discussed in a team environment involving the medical oncologist, pathologist, radiologist, and radiation oncologist in addition to the surgeon. There were some minimum multidisciplinary case numbers recommended by disease site, as shown in Table 5. The need to develop surgeons who could lead these conferences was part of the core rationale for creating the CGSO Board as a separate component board of the ABS.

The discussion around case numbers and recommending an increase was thought to be critical to the training of a surgical oncologist and paralleled the milestones that were developed by the ACGME and ABS for the fellows within accredited CGSO fellowships. The CGSO Board recognized that fellows may have variable control over their individual operative exposure, and thus minimal numbers should be programmatic requirements for accreditation of Complex General Surgical Oncology training programs by the RRC-S. An initial motion supporting the new case numbers and case list was passed by the CGSO Board on January 10, 2015, and by the ABS on January 14, 2015. The CGSO board then worked with the RRC-S to slightly modify the case number and provide more clear-cut definitions, which are described above and outlined in Table 5. These minor revisions were then reviewed and approved by both CGSO Board and the ABS at the June retreat in 2015. The target goal for the new case numbers will apply to fellows starting CGSO fellowships in August of 2016.

The CGSO Board is currently working with the ACGME to update the CGSO case log to reflect various complex surgical oncology procedures as well as to develop categories for multivisceral and multidisciplinary procedures.

Currently there are 22 accredited programs in Complex General Surgical Oncology, with 57 positions for training available in these programs. In reviewing the case numbers from individuals applying for certification, the 240 case number would be achieved by $92.1 \%$ of individuals completing training in $2013(n=38)$ and $94.4 \%$ of individuals completing training in $2014(n=36)$. The types and ranges of individual cases performed varies widely among programs, but the distribution of case numbers generally parallels the distribution recommended by the CGSO Board as outlined in Table 5.

\section{THE FUTURE}

Although much has been achieved with creation of a new specialty board certificate, much remains to be done.
The CGSO Board is working to optimize a SCORE curriculum for CGSO fellows and training programs called SO-SCORE. Given that the new certificate is time limited to 10 years, the CGSO board has just started to discuss what the maintenance of certification program and recertification process for individuals who become certified should look like. A potentially challenging problem relates to the fact that many of the individuals who obtain this certificate will go onto develop a subspecialized practice in surgical oncology such as breast or hepatobiliary. The concept of a modular recertification process is one that the ABS is currently wrestling with in general surgery but that may also need to be developed for the recertification of CGSO diplomats, depending how the practices of this group of surgeons evolves over time. Finally, there are discussions currently underway within the ABMS to determine if there should be certificates of focused expertise granted by the ABS for recognizing additional training and competence in non-ACGME-approved fellowships. This may involve the CGSO Board, as it could pertain to surgical fellowships that have a heavy cancer focus, like breast fellowships, endocrine fellowships, and hepatobiliary fellowships. As Dr. Balch mentioned during the delivery of the Ewing Lecture at the 2015 annual cancer symposium of the SSO, "Specialty board recognition was an original strategy of the Founding Fathers and continued to be one of the top agenda items throughout our 75-year history." 4 Indeed, recognition of cancer surgery as a specialty was one of the objectives mentioned in the James Ewing Society Board minutes as early as 1946. Thus, to a certain extent, the first cadre of certified surgical oncologists represents both the successful reaching of the finish line and the beginning of a new era for surgical oncology.

DISCLOSURE The authors declare no conflict of interest.

\section{REFERENCES}

1. Michelassi F. Subspecialty certificate in advanced surgical oncology (SSO presidential address). Ann Surg Oncol. 2010;17:3094103.

2. Michelassi F. American Board of Surgery certificate in complex general surgical oncology: a reality. Ann Surg Oncol. 2011;18: 2405-6.

3. Michelassi F. Update on the American Board of Surgery subspecialty certificate in complex general surgical oncology. Ann Surg Oncol. 2013;20:2013-4.

4. Balch CM, Coit D, Berman RS. The 75-year history of the Society of Surgical Oncology — part 1: the traditional years (1940-1965) (2015 James Ewing Lecture). Ann Surg Oncol. 2015. Available at: http://www.surgonc.org/docs/default-source/pdf/sso-hx-part-i-asosept-2015.pdf?sfvrsn=4. Accessed January 27, 2016. 